\title{
Substation DC system grounding fault prediction method
}

\author{
Guozhong Wang ${ }^{1 *}$ \\ ${ }^{1}$ Wenshan Power Supply Bureau of Yunnan Power Grid Co., Ltd., Wenshan, Yunnan, 663000, China
}

\begin{abstract}
There may be disturbance and uncertainty in the collection of leakage current in DC system of substation, which leads to the decrease of accuracy and increase of prediction error. Based on this, an improved grey prediction method is proposed to predict DC system branch grounding fault. Firstly, the characteristics of DC system ground fault parameters are collected. Secondly, the improved grey prediction algorithm is used to predict and estimate whether the detection reaches the fault threshold in the future. Finally, the validity of the proposed method is verified by MATLAB modeling.
\end{abstract}

\section{Introduction}

After the grounding fault is detected in the current DC system, it is still necessary to find the fault point manually or to judge the specific fault location by relying on the unique experience of a certain person. On the one hand, it is impossible to predict the fault in advance before the fault occurs. After the failure occurs, the expert resources will also be strained. On the other hand, due to insufficient information, the investigation is more difficult. Therefore, it is necessary to introduce an efficient intelligent method for fault diagnosis and prediction.

According to the different prediction methods, the current fault prediction mode can be roughly divided into three types: prediction method based on time migration, prediction method based on early micro signal detection and prediction method based on qualitative analysis [1]. Since the main parameter used in DC system branch grounding fault detection is the leakage current of each branch, only the past and present parameters can be referred. Therefore, the prediction method based on time migration is selected. The prediction method based on time migration mainly analyses the change trend of relevant data in the past time of the system to be tested. Combined with the current situation, the future state of the system to be tested is estimated, so as to judge whether the system under test will fail. It can be subdivided into prediction methods based on classical time series analysis, grey model theory and filter [2-3]. In the classical time series analysis method, the obtained state data sequence is regarded as a random sequence. Using the dependence between adjacent observation values, the time series is fitted by establishing a mathematical model. Because most of the data models used for prediction are linear models or their improvement models, the effect for nonlinear systems is not very ideal [4]. Grey model theory is a mathematical method proposed by Deng Julong to solve the problem of poor information system (grey system).
Because most of the systems in real production and life are grey systems with few parameters that can be directly observed. This prediction method has been widely used in various fields [5-6]. However, the type of DC system grounding fault is complex, and the reference parameters are less, and the prediction error of traditional grey prediction method is large.

Therefore, an improved grey prediction method is proposed in this paper. Firstly, the basic principle of grey prediction is analysed, and then an improved grey prediction method is proposed to improve the accuracy of branch grounding fault prediction. Finally, the effectiveness of the proposed method is verified by simulation results.

\section{DC system grounding fault prediction algorithm}

\subsection{Analysis of grey prediction principle}

The basic principle of the traditional grey prediction model is to process the original data and establish the grey model. Master the development law of the system, and make a scientific quantitative prediction of the future state of the system. The core of grey prediction model is to transform historical data with time into differential equation. In order to establish a dynamic grey prediction model which can predict the development trend of the system.

Suppose the original nonnegative sequence data sequence.

$$
X^{(0)}(i)+a Z^{(1)}(i)=b
$$

where $X^{(0)}(i)$ is the original sequence, a is the development coefficient, b is the grey dosage, $Z^{(1)}(i)$ is the albino background sequence;

$X^{(I)}(i)$ is a cumulative generating sequence of original

\footnotetext{
"Corresponding author's e-mail: zhoudan@zjut.edu.cn
} 
data $X^{(0)}(i)$.

$$
\begin{aligned}
& X^{(1)}(k)=\sum_{i=1}^{k} x^{(0)}(i) \\
& k=1,2 \cdots, n
\end{aligned}
$$

After the original sequence with strong randomness is accumulated and generated, the influence of bad data in the original sequence $X^{(0)}(i)$ is weakened, and the generated sequence has exponential growth law. Therefore, the whitening equation of model GM $(1,1)$ can be constituted by a first-order differential equation containing only one variable.

$$
\frac{d x^{(1)}}{d t}+a x^{(1)}=b
$$

The grey prediction model is obtained by discretizing the whitening equation.

$$
x^{(0)}(k)+a z^{(1)}(k)=b
$$

The solution is obtained:

$$
\hat{x}^{(1)}(k+1)=\left(x^{(0)}(1)-\frac{b}{a}\right) e^{-a k}+\frac{b}{a}
$$

where $-\mathrm{a}$ is the development coefficient, reflecting the development trend; B is the grey action quantity, which is the data representing the development and change law of the original data series.

The parameters to be identified in equation (5) are solved by least square method.

$$
\hat{\boldsymbol{a}}=[\mathrm{a}, \mathrm{b}]^{T}=\left(\boldsymbol{B}^{T} \boldsymbol{B}\right)^{-1} \boldsymbol{B}^{T} \boldsymbol{Y}
$$

where

$$
\begin{gathered}
\boldsymbol{B}=\left[\begin{array}{cc}
-\frac{1}{2}\left(X^{(1)}(1)+X^{(1)}(2)\right) & 1 \\
\vdots & \vdots \\
-\frac{1}{2}\left(X^{(1)}(n-1)+X^{(1)}(n)\right) & 1
\end{array}\right]=\left[\begin{array}{cc}
-Z^{(1)}(2) & 1 \\
\vdots & \vdots \\
-Z^{(1)}(n) & 1
\end{array}\right] \\
\boldsymbol{Y}=\left[x^{(0)}(2), x^{(0)}(3), \cdots, x^{(0)}(n)\right]^{T}
\end{gathered}
$$

By reducing formula (5), we can get the prediction formula of the original series grey model.

$$
\begin{aligned}
& \hat{x}^{(0)}(k+1)=\hat{x}^{(1)}(k+1)-\hat{x}^{(1)}(k) \\
& =\left(1-e^{a}\right)\left(x^{(0)}(1)-\frac{b}{a}\right) e^{-a k}
\end{aligned}
$$

where $k=1,2, \cdots, n$.

\subsection{Branch grounding fault prediction}

The main parameters used in DC system branch grounding fault detection are the voltage of positive and negative bus to ground and leakage current of each branch. Because there are few types of parameters that can be directly observed, it belongs to "poor information" system. Therefore, it is considered to use grey prediction algorithm to analyse the trend of past and present data. Forecast and estimate the future situation to see whether the fault threshold is reached, so as to judge whether the DC system branch is likely to fail. There are many reasons for DC system grounding fault.

In addition, there may be disturbance and uncertainty in the collection of leakage current in actual substation environment. The accuracy of fault prediction is reduced and the prediction error is increased. Therefore, on the basis of the conventional fitting model, the original data series are smoothed by trigonometric transformation and converted into new series. Then, the new series is fitted with $y^{(1)}(t)$ which is generated by the sum of exponential equation $Y=\mathrm{ae}^{x}$ and linear regression equation $Y=\mathrm{aX}+\mathrm{b}$. The grey prediction algorithm is used to calculate the prediction sequence, and finally the prediction series is restored to the original series.

The specific implementation process is as follows: firstly, extract the historical data samples of $N$ points and do exponential smoothing to get the new sequence, as shown in the formula.

The original $X^{(0)}=\left(X^{(0)}(1), X^{(0)}(2), \cdots, X^{(0)}(n)\right)$ sequence sequence after function transformation: be the $Y^{(0)}=\left(y^{(0)}(1), y^{(0)}(2), \cdots, y^{(0)}(n)\right)$ where $y^{(0)}(t)=\operatorname{arcot}\left(x^{(0)}(t)\right)^{a}$

The time response formula of GM $(1,1)$ model is denoted as:

$$
\hat{y}^{(1)}(t+1)=C_{1} e^{v t}+C_{2}
$$

The sum of the exponential equation $Y=\mathrm{ae}^{x}$ and the linear regression equation $Y=\mathrm{aX}+\mathrm{b}$ was used to fit the $y^{(1)}(t)$.

$$
\hat{y}^{(1)}(t)=C_{1} e^{v t}+C_{2} t+C_{3}
$$

The value of $C_{1}, C_{2}, C_{3}$ and $\mathrm{v}$ is calculated as follows.

Suppose the parameter sequence

$$
\begin{aligned}
Z(t) & =\hat{X}^{(1)}(t-1)-\hat{X}^{(1)}(t) . \\
& Z(t)=\hat{X}^{(1)}(t-1)-\hat{X}^{(1)}(t) \\
& =C_{1} e^{v(t+1)}+C_{2}(t+1)-C_{1} e^{v t}-C_{2} t-C_{3} \\
& =C_{1} e^{v t}\left(e^{v}-1\right)+C_{2} \\
& t=1,2, \cdots, n-1
\end{aligned}
$$

The parameter sequence $Y_{m}(t)=Z(t+m)-Z(t)$.

$$
\begin{aligned}
& Y_{m}(t)=Z(t+m)-Z(t) \\
& =C_{1} e^{v(t+m)}\left(e^{v}-1\right)+C_{2}-C_{1} e^{v t}\left(e^{v}-1\right)-C_{2} \\
& \quad Y_{m}(t+1)=C_{1} e^{v(t+1)}\left(e^{v m}-1\right)\left(e^{v}-1\right)
\end{aligned}
$$

The solution of parameter $\mathrm{v}$ can be obtained by taking the ratio of $Y_{m}(t+1)$ and $Y_{m}(t)$.

$$
Y_{m}(t+1) / Y_{m}(t)=\mathrm{e}^{v}
$$




$$
\mathrm{V}=\ln \left[Y_{m}(t+1) / Y_{m}(t)\right]
$$

The estimated values of parameters $\mathrm{C} 1, \mathrm{C} 2$ and $\mathrm{C} 3$ can be obtained by using the least square method.

$$
\boldsymbol{X}^{(1)}=\left[\begin{array}{c}
X^{(1)}(1) \\
X^{(1)}(2) \\
\vdots \\
X^{(1)}(n)
\end{array}\right], \boldsymbol{C}=\left[\begin{array}{c}
C_{1} \\
C_{2} \\
C_{3}
\end{array}\right], \boldsymbol{A}=\left[\begin{array}{lll}
e^{v t} & 1 & 1 \\
e^{v t} & 2 & 1 \\
\vdots & \vdots & \vdots \\
e^{v t} & n & 1
\end{array}\right]
$$

where $\boldsymbol{X}^{(1)}=\boldsymbol{A} \boldsymbol{X}$,the estimated values of parameters $\mathrm{C} 1, \mathrm{C} 2$ and $\mathrm{C} 3$ can be obtained by inverse transformation.

$$
\boldsymbol{C}=\left(\boldsymbol{A}^{T} \boldsymbol{A}\right)^{-1} \boldsymbol{A}^{T} \boldsymbol{X}^{(1)}
$$

By substituting the values of parameters $\mathrm{C} 1, \mathrm{C} 2$ and $\mathrm{C} 3$ and $\mathrm{v}$ into formula (12), the predicted values of the transformed cumulative generation sequence can be obtained.

The prediction value of the original sequence after transformation can be obtained by the reduction and reduction.

$$
\hat{y}^{(0)}(t+1)=\hat{y}^{(1)}(t+1)-\hat{y}^{(1)}(t)
$$

The flow chart of the improved grey prediction algorithm is shown in Figure 1.

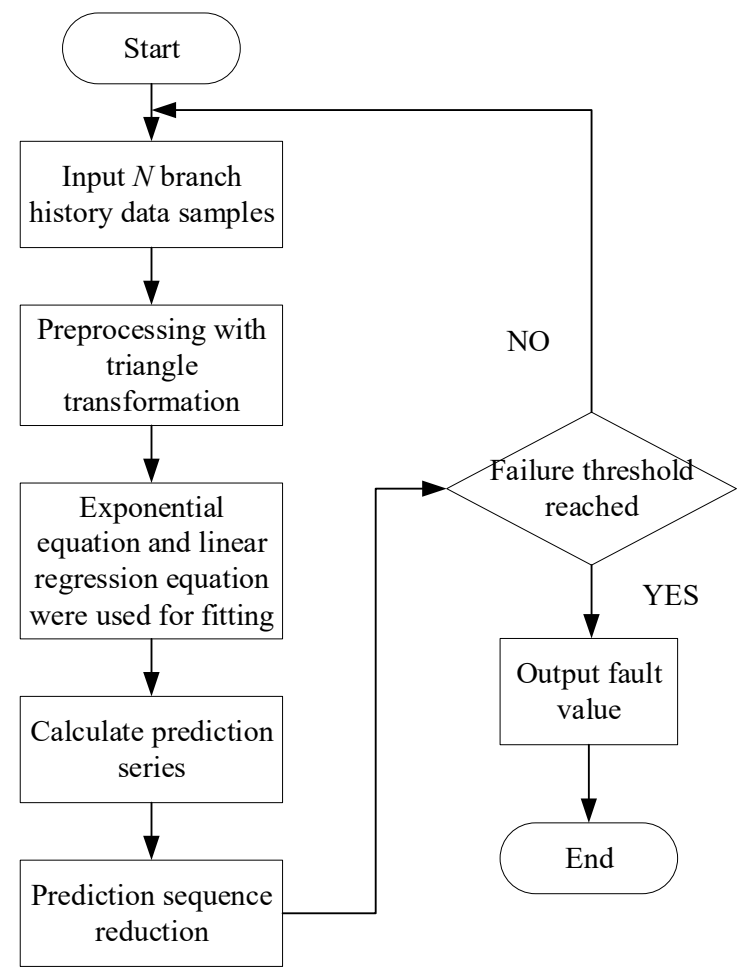

Figure 1. DC system branch grounding fault prediction algorithm flow

\section{Case study}

\subsection{Branch ground fault}

The sample data of 10 consecutive acquisition times of the branch are selected. The traditional grey prediction model and the improved grey prediction model are established respectively, and the traditional and improved prediction values are obtained. Draw the actual data and the predicted data in the two ways on the same curve, and get the comparison curve as shown in Figure 2. The last four predicted values are compared with the actual data values. The data of 10 samples are shown in Table 1.

Table 1. Branch history data

\begin{tabular}{ccccc}
\hline $0.64 \mathrm{ma}$ & $1.25 \mathrm{ma}$ & $1.45 \mathrm{ma}$ & $1.52 \mathrm{ma}$ & $1.79 \mathrm{ma}$ \\
& & & & \\
\hline $2.02 \mathrm{ma}$ & $2.38 \mathrm{ma}$ & $2.49 \mathrm{ma}$ & $2.65 \mathrm{ma}$ & $2.95 \mathrm{ma}$ \\
\hline
\end{tabular}

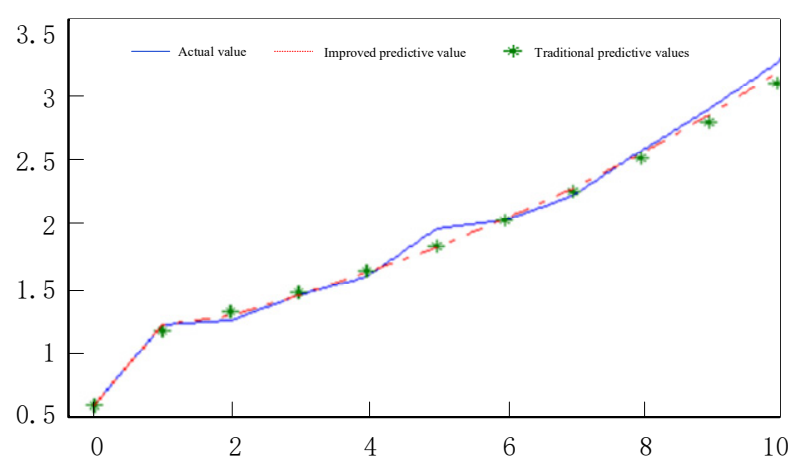

Figure 2. Comparison of fault prediction

The prediction results of the last four data can be obtained by using the relative error calculation formula, as shown in Table 2.

Table 2. Comparison of prediction results

\begin{tabular}{ccccc}
\hline numbers & 7 & 8 & 9 & 10 \\
\hline $\begin{array}{c}\text { actual value } \\
\text { Traditional } \\
\text { predictive } \\
\text { values }\end{array}$ & $2.38 \mathrm{ma}$ & $2.49 \mathrm{ma}$ & $2.65 \mathrm{ma}$ & $2.95 \mathrm{ma}$ \\
$\begin{array}{c}\text { Improved } \\
\text { predictive } \\
\text { value }\end{array}$ & $2.39 \mathrm{ma}$ & $2.45 \mathrm{ma}$ & $2.61 \mathrm{ma}$ & $2.90 \mathrm{ma}$ \\
$\begin{array}{c}\text { Traditional } \\
\text { prediction } \\
\text { bias }\end{array}$ & $0.84 \%$ & $1.61 \%$ & $2.63 \mathrm{ma}$ & $2.93 \mathrm{ma}$ \\
$\begin{array}{c}\text { Improved } \\
\text { prediction } \\
\text { bias }\end{array}$ & $0.42 \%$ & $0.40 \%$ & $0.75 \%$ & $0.68 \%$ \\
\hline
\end{tabular}

It can be seen that the two prediction methods can better follow the actual data when the prediction interval is small. In addition, the prediction accuracy of the improved grey prediction method is higher than that of the traditional grey prediction method.

\section{Conclusion}

In this paper, an improved grey prediction method is proposed to reduce the accuracy and increase the prediction error of DC system branch grounding fault in substation. Firstly, the characteristics of DC system grounding fault parameters are collected. Secondly, the original data series are transformed into new series by 
trigonometric transformation, and then the exponential equation and linear equation are used for the new sequence Finally, the prediction series is restored to the predicted value of the original series. On this basis, the improved grey prediction algorithm is proved to be more accurate than the traditional grey prediction algorithm on shorter data sequence by MATLAB modeling.

\section{References}

1. Zhou, B.H., Li,F.T., Song,X.F.(2019) Commutation Failure Prediction and Control System Optimization Based on DC Current Variation. Power System Technology, 43:3497-3503

2. Chen,Y.H., Yang. B., Dong, J. W.(2006) Time-series prediction using a local linear wavelet neuralnetwork. Neurocomputing, 69: 449-465.

3. Li, D.C., Chang,C.J.(2009) An improved grey-based approach for early manufacturing data forecasting. Computer \& Industrial Engineering, 57:1161-1167.

4. Phinikarides A, Makrides G, Zinsser B, et al.(2015) Analysis of photovoltaic system performance time series: Seasonality and performance loss. Renewable Energy, 77:51-63.

5. Niu, D.X., Zhang, B., Meng, M.(2006) Grey Model of Power Load Forecasting Based on Particle Swarm Optimization. In: Proceeding of 6th Worm Congress on Intelligent Control and Automation, Dalain, China, pp: 7651-7655.

6. Li, J. F, Yang, A. P. (2007) New Approach of Building GM(I,1) Background Value and Its Application. In: Proceedings of the IEEE International Conference on Automationg and Logistics, Shenyang, China, pp: 1619. 